\title{
DESCRIPCIÓN PRELIMINAR DE LAS LLAMADAS DE ANUNCIO DE ELEUTHERODACTYLUS PATRICIAE (ANURA: ELEUTHERODACTYLIDAE), EN EL PARQUE NACIONAL VALLE NUEVO, REPÚBLICA DOMINICANA
}

\section{Preliminary description of the advertisement calls of Eleutherodactylus patriciae (Anura: Eleutherodactylidae) in Valle Nuevo National Park, Dominican Republic}

\author{
Cristian F. Marte-Pimentel ${ }^{1}$, Luis M. Díaz ${ }^{2}$ y Sixto J. Incháustegui ${ }^{3}$
}

\begin{abstract}
'Museo Nacional de Historia Natural "Prof. Eugenio de Jesús Marcano", Santo Domingo, República Dominicana. (D) orcid.org/0000-0002-2159-8238. ${ }^{2}$ Museo Nacional de Historia Natural de Cuba, Obispo \#61, Esquina a Oficios, Habana Vieja. (1) orcid.org/0000-0002-7213-7239, luisfromcuba@yahoo.es. ${ }^{3}$ Grupo Jaragua. El Vergel \#33, El Vergel, Santo Domingo, República Dominicana. (1) orcid.org/0000-0002-7135-0871, sixtojinchaustegui@yahoo.com.

Para correspondencia: c.marte@mnhn.gov.do
\end{abstract}

[Recibido: 15 de abril, 2021. Aceptado para publicación: 13 de diciembre, 2021]

\section{RESUMEN}

La mayoría de las especies del género Eleutherodactylus de La Hispaniola carecen de descripciones cuantitativas de sus llamadas de anuncio. Entre tales especies se encuentra E. patriciae, una rana endémica de la Cordillera Central de la cual solo se tenía una referencia onomatopéyica de sus vocalizaciones. Se analizaron las grabaciones de 8 machos de E. patriciae en dos sabanas de pajón (Danthonia domingensis) dentro del Parque Nacional Valle Nuevo, para cada uno de los cuales se midieron siete variables temporales y dos variables espectrales en 20 llamadas respectivas. Las llamadas de anuncio son silbidos con una duración promedio de 0.4 segundos, una tasa de repetición promedio de 16.6 señales/minuto, y una frecuencia dominante promedio de $2.1 \mathrm{kHz}$. Cada llamada tiene una estructura armónica que se extiende al espectro ultrasónico $(>20 \mathrm{kHz})$.

Palabras clave: análisis bioacústico, variables espectrales, variables temporales, subgénero Eleutherodactylus, Hispaniola.

\section{ABSTRACT}

Most of the Hispaniolan species of the genus Eleutherodactylus lack quantitative descriptions of their advertisement calls. Among such species is E. patriciae, an endemic frog of the Central Cordillera of which there was only an onomatopoeic reference of its vocalizations. We recorded 8 males of E. patriciae in two savannas covered by "pajón" (Danthonia domingensis) in the Valle Nuevo National Park. For each call, we measure seven temporal variables and two spectral variables in 20 respective calls. Advertisement calls are whistles with 0.4 seconds mean duration, a mean repetition rate of 16.6 signals / minute, and a mean dominant frequency of $2.1 \mathrm{kHz}$. Each call has a harmonic structure that extends to the ultrasonic spectral range $(>20 \mathrm{kHz})$.

Keywords: bioacoustic analysis, spectral variables, temporal variables, subgenus Eleutherodactylus, Hispaniola. 


\section{INTRODUCCIÓN}

En La Hispaniola, los anfibios (76 especies) constituyen el segundo grupo de vertebrados con mayor número de especies endémicas (98\%), destacándose el género Eleutherodactylus (Familia Eleutherodactylidae), con 66 especies, todas endémicas. La radiación adaptativa del género ha permitido que se halle representado en una amplia diversidad de ecosistemas, desde los bosques secos hasta las mayores altitudes (Hedges et al., 2008). La mayoría de las especies tienen un alto endemismo local, por lo que la pérdida, fragmentación y deterioro de las condiciones de los hábitats aumenta el riesgo de extinción de estas especies (Pough, 1999; Rueda, 1999; Urbina-Cardona y Pérez-Torres, 2002). Las especies de alta montaña pueden estar entre las más afectadas no solo por las modificaciones antropogénicas del hábitat sino también por el cambio climático global (Foden et al., 2018).

En términos herpetológicos, la Cordillera Central posee uno de los mayores niveles de endemismos conocidos para la República Dominicana, por lo que merecen mucha atención. Uno de los ecosistemas altitudinales más característicos en este sistema montañoso es la sabana de pajón (1350-2950 msnm), son zonas abiertas de gran extensión, suelos muy húmedos y vegetación baja, se encuentran dominadas casi exclusivamente por la especie Danthonia domingensis, la cual es conocida comúnmente como "Pajón", estas sabanas son de suma importancia y un gran número de ellas se encuentran en el Parque Nacional Valle Nuevo (Cassá, 2012). Hasta el momento, estas formaciones vegetales no han sido debidamente consideradas como unidades de vegetación. Hager y Zanoni (1993), incluyen las sabanas como un subtipo de vegetación relacionada al bosque de pinos denominada "sabanas de las montañas altas". Ante la falta de información relacionada con la herpetofauna asociada al suelo en el ecosistema de sabanas, algunos trabajos resaltan la necesidad de estudiarlas, debido a las peculiaridades que poseen (Núñez et al., 2006), entre las que podríamos destacar que constituyen una fuente importante de agua y la amplia disponibilidad de microhábitats que pueden albergar (Frolich et al., 2003; Guerrero y McPherson, 2002). Conocer aspectos de la ecología de las especies que habitan en estos tipos de vegetación nos ayudará a tomar mejores decisiones para futuros planes de conservación.

La especie Eleutherodactylus patriciae Schwartz es endémica de la Cordillera Central. Es una de las representantes del género que vive a mayor altitud en el Caribe insular, por encima de los 2000 metros (Fig. 1). Es una especie terrestre que vive en bosques de pinos y bosques latifoliados. De día se suele encontrar debajo de troncos, rocas y en zonas alteradas debajo de maderas, césped y objetos humanos (Schwartz y Henderson, 1991). Es moderadamente común en hábitats adecuados (Hedges et al., 2004; Stuart et al., 2008). Los machos pueden medir $29 \mathrm{~mm}$ y las hembras hasta $35 \mathrm{~mm}$ de longitud hocico-cloaca (Schwartz, 1964). La mayor actividad acústica de E. patriciae se ha registrado en la noche, cuando los machos se trasladan a la parte alta de la vegetación buscando perchas para vocalizar (Henderson y Powell, 2009). Los machos producen sus llamadas desde el suelo, rocas y la vegetación baja (arbustos, helechos), hasta un metro de altura. Las puestas contienen entre 18-24 huevos y presentan desarrollo directo (Hedges et al., 2004; Stuart et al., 2008). Esta especie se encuentra bajo la categoría En Peligro (EN), tanto en la Lista Roja de la Unión Internacional para la Conservación de la Naturaleza (UICN, 2014) como en la Lista Roja Nacional del Ministerio de Medio Ambiente y Recursos Naturales (Ministerio de Medio Ambiente y Recursos Naturales, 2011). 


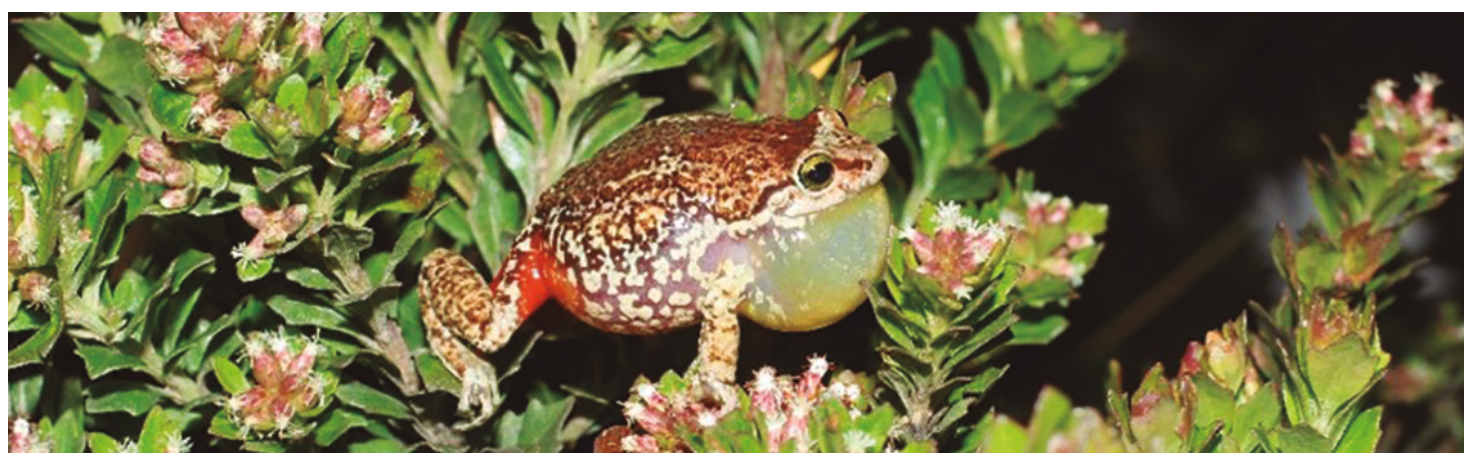

Figura 1. Eleutherodactylus patriciae (Schwartz, 1964), individuo macho vocalizando.

La generalizada falta de información acerca de la ecología y conducta de las especies del género Eleutherodactylus, unido al alto riesgo de extinción que tienen sus poblaciones, hace necesario centrar las investigaciones en esos aspectos, ya que resultan de suma importancia para trazar estrategias de conservación adecuadas (Granda-Rodríguez et al., 2008; Sangermano et al., 2015a, b). La comunicación acústica de los anfibios desempeña una función crucial para la reproducción y delimitación de territorios (Wells, 2007), y es utilizada como herramienta en sistemática, conservación y en manejo de poblaciones naturales (Köhler et al., 2017). Uno de los aspectos más interesantes de la conducta de los anfibios es el repertorio vocal, las emisiones acústicas de estos animales son importantes para su identificación, ya que en la mayoría de los casos son especie-específicos (Díaz y Cádiz, 2008).

Por lo general, las especies de la serie auriculatus, grupo varians, del subgénero Eleutherodactylus (sensu Hedges et al., 2008), son predominantemente arborícolas y suelen producir vocalizaciones audibles en la distancia, debido a su intensidad. La presencia de armónicos ha sido registrada en otras especies de Eleutherodactylus de La Hispaniola (Galvis et al. 2016a) y Puerto Rico (Ríos-Franceschi y Joglar, 2016), sin que exista una aparente explicación funcional, estudiar el espectro no audible de las vocalizaciones, resulta de interés para estudios conductuales de los anfibios.

\section{OBJETIVOS}

- Describir por primera vez algunas de las características espectrales y temporales de las llamadas de anuncio de Eleutherodactylus patriciae.

- Caracterizar el entorno ecológico al cual se asocian los machos durante su actividad acústica en las sabanas de pajón.

\section{MATERIALES Y MÉTODOS}

Área de estudio. El Parque Nacional Valle Nuevo se encuentra localizado en la Cordillera Central de la República Dominicana (Fig. 2). Fue declarado área protegida en el año 1983. Posee un área aproximada de $910 \mathrm{~km}^{2}$ y está ubicado entre las provincias Monseñor Nouel, La Vega, Azua y San José de Ocoa. Este parque posee una cobertura boscosa aproximada de $390 \mathrm{~km}^{2}$, de los cuales los bosques de pinos abarcan la mayor extensión con $244045 \mathrm{~km}^{2}$, 
entre los bosques de coníferas (pinos) se encuentran las sabanas de montañas altas (sabanas de pajón), dominadas casi exclusivamente por la especie endémica Danthonia domingensis, seguidos por los bosques nublados, con $124.5 \mathrm{~km}^{2}$, parches de bosques latifoliados que cubren una extensión de $16.3 \mathrm{~km}^{2}$ y por último pequeños parches cubiertos por manaclas (Prestoea montana), (Guerrero y McPherson, 2002). Las áreas donde fueron realizadas las grabaciones y observaciones ecológicas, corresponden a las sabanas conocidas como Sabana de los Frailes o La Lechuga (La Pirámide), en las coordenadas $18^{\circ} 42^{\prime} 25^{\prime \prime} \mathrm{N} ; 7^{\circ} 36^{\prime} 11^{\prime \prime} \mathrm{O}$, y

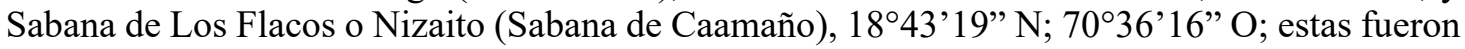
escogidas por ser sabanas conservadas, no afectadas por fuegos o actividades agrícolas, cada localidad fue visitada durante una temporada lluviosa y una temporada seca, en los meses de junio-agosto y enero, respectivamente.

A
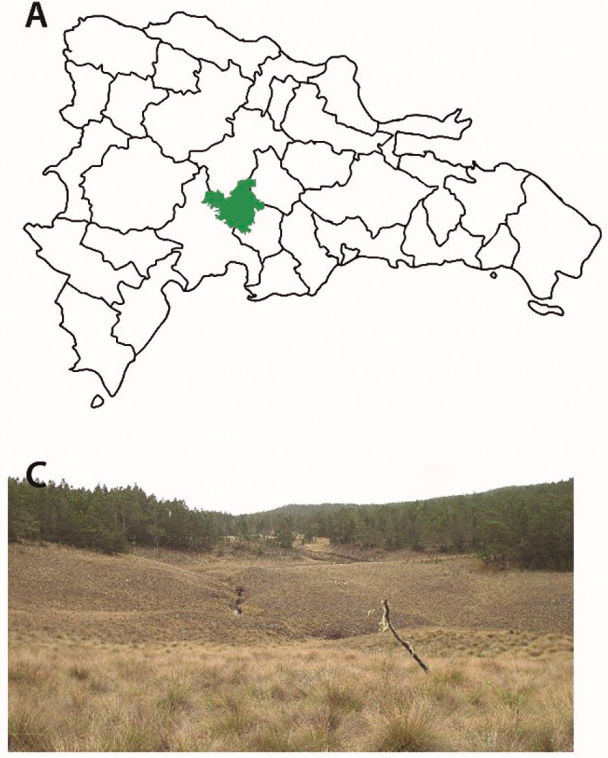
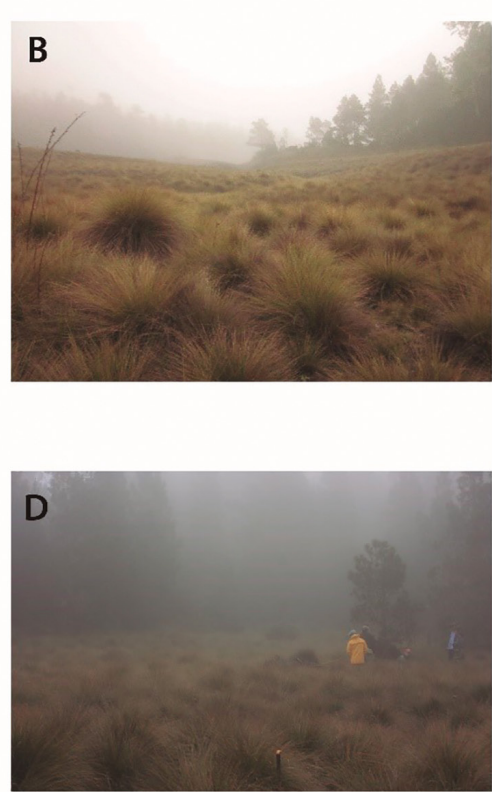

Figura 2. Localidades de observación y registro. A, delimitación del Parque Nacional Valle Nuevo; B-C, vistas de la sabana Los Flacos, o Nizaíto (sabana de Caamaño); D, sabana de los Frailes (La Pirámide).

Grabación de las llamadas de anuncio. Las grabaciones se realizaron durante la noche, entre las 20:30 y 1:30 horas. El equipo de grabación fue una grabadora Marantz 660 y un micrófono unidireccional Sennheiser ME 66. Las grabaciones se realizaron con una frecuencia de muestreo de $44 \mathrm{kHz}$ y un tamaño de muestra de 32 bits. Los individuos fueron grabados a una distancia de $0.5-2 \mathrm{~m}$. La descripción acústica se basó en las grabaciones de ocho individuos de E. patriciae en dos sabanas de pajón (Danthonia domingensis) dentro del Parque Nacional Valle Nuevo, para cada uno de los cuales se midieron siete variables temporales y dos variables espectrales en 20 llamadas. Los ejemplares testigos (Vouchers) fueron depositados en la colección herpetológica del Museo Nacional de Historia Natural "Profesor Eugenio de Jesús Marcano", Santo Domingo, República Dominicana, bajo los números calcográficos MNHNSD 23.4040-4047. Las grabaciones se acompañaron con la descripción cualitativa del hábitat y condiciones ambientales, las cuales fueron tomadas con el medidor meteorológico portátil Kestrel 3000. Las temperaturas máxima y mínima fueron medidas usando un termómetro digital modelo SN 89212, marca Forestry Suppliers; para el monitoreo de la temperatura a lo largo de dos días $\mathrm{y}$ tres noches fueron utilizados los medidores tipo Ibutton Termometer. 
Análisis bioacústico. Las variables temporales y espectrales de las emisiones acústicas fueron medidas con el programa Raven Pro 1.4 (Cornell Lab of Ornithology; Ithaca, New York, EEUU). El valor de DFT (Transformada Rápida de Fourier; =FFT, Fast Fourier Transform) para generar los espectrogramas fue de 512 puntos, en ventanas tipo Hanning. Para la caracterización de las llamadas de anuncio se utilizaron las mediciones espectrales y temporales descritas por Köhler et al. (2017) y Díaz et al. (2018).

Las variables temporales medidas fueron las siguientes (en el oscilograma): (1) duración de la llamada (tiempo comprendido entre el inicio y el final de la llamada), (2) período de llamadas (tiempo entre el inicio de la primera llamada y el inicio de la siguiente), (3) duración de las notas (tiempo comprendido entre el inicio y el final de la nota), (4) intervalo entre notas (espacio comprendido entre el final de una nota y el inicio de la otra), (5) tasa de repetición de las llamadas (número de llamadas por minuto), (6) número de notas por llamada (número de notas por tren de llamadas) y (7) tasa de repetición de notas (número de notas por segundo). Las variables espectrales medidas fueron (en el espectro de potencia): (8) frecuencia dominante (frecuencia donde se halla el pico de máxima intensidad del sonido), y (9) ancho de banda $\Delta \mathrm{F}$ a un umbral -10 dB por debajo del pico de máxima energía. Para la tabulación de los datos y la extracción de los promedios se utilizó el programa Microsoft Excel.

\section{RESULTADOS}

Caracterización de las llamadas de anuncio. Cada llamada de anuncio de E. patriciae se escucha como un silbido espaciado, las emisiones constan de una única nota, tienen una duración de $0.2-0.5(\bar{x}=0.4)$ segundos y se repiten a razón de 14-21 ( $\bar{x}=16.6)$ señales/minuto. El periodo de llamadas es 2.3-11.2 ( $\overline{\mathrm{x}}=3.8)$ segundos. La frecuencia dominante es $1.9-2.4(\overline{\mathrm{x}}=2.1) \mathrm{kHz}$ y coincide con la frecuencia fundamental, pero se definen armónicos que abarcan una gama espectral entre 2.1 y $23.9 \mathrm{kHz}$. Las llamadas incrementan su intensidad gradualmente hasta un pico máximo de amplitud que se alcanza en la mitad posterior de la llamada, desde donde disminuye para terminar abruptamente (Fig. 3). La temperatura ambiental bajo la cual los individuos fueron grabados fue $12-26^{\circ} \mathrm{C}\left(\overline{\mathrm{x}}=16^{\circ} \mathrm{C}\right)$.

Notas sobre el microhábitat. Las observaciones fueron realizadas en las sabanas dominadas por la especie (Danthonia domingensis). En el área de estudio predominó la vegetación arbustiva tupida y las plantas herbáceas, con alturas comprendidas entre 0 y 0.6 metros sobre el suelo, con presencia de árboles jóvenes entre 0.6 y $3.0 \mathrm{~m}$ de altura. La densidad de pajones (Danthonia domingensis) es alta, 1-2 individuos maduros por metro cuadrado, lo cual se traduce a una cobertura del suelo de $80-100 \%$.

Notas ecológicas. La mayor actividad acústica de Eleutherodactylus patriciae se registró durante la temporada de lluvias. En la seca, la actividad fue baja y se escucharon pocos machos vocalizando. Durante el día, el $90 \%$ de los individuos registrados se encontraron ocultos en la parte interna del pajón, mientras que durante la noche los individuos fueron registrados expuestos en la parte superior de las plantas. Se registraron todas las clases de edades (adultos, 14; juveniles, 21 y puestas, 2), siendo los juveniles proporcionalmente los más frecuentes en los muestreos realizados durante la temporada de lluvias (21), comparados con los adultos (10). Se observaron dos puestas en esta temporada. Durante la temporada seca solo fueron registrados individuos adultos (4). 
A
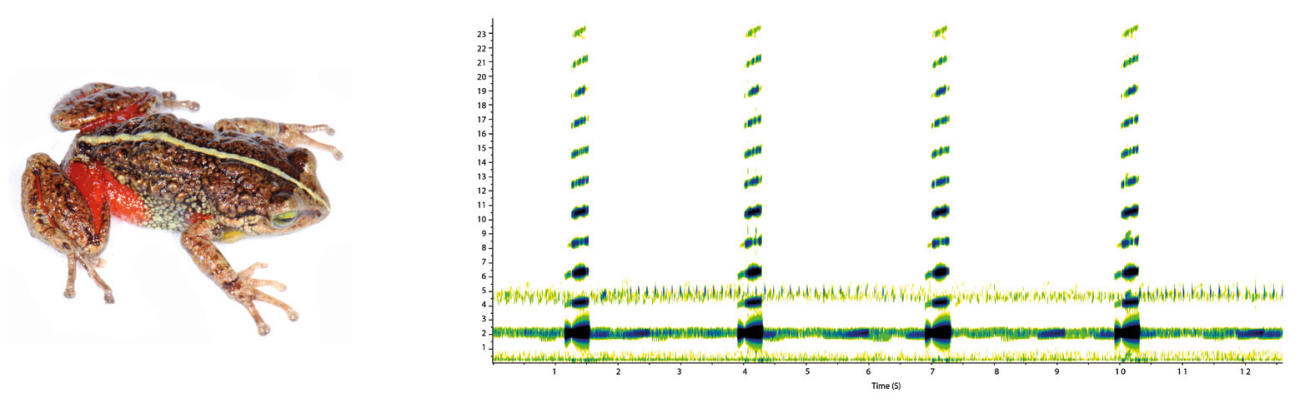

B
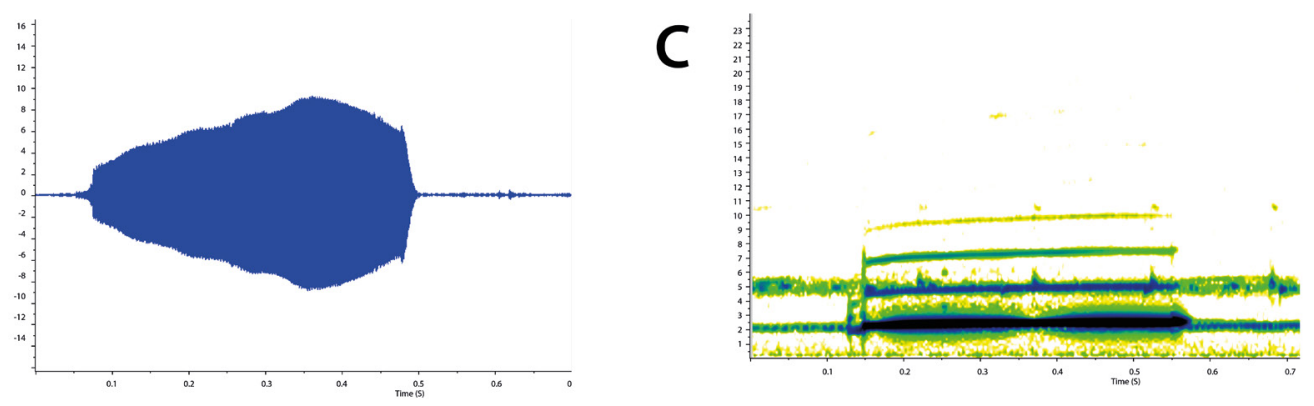

Figura 3. Oscilograma y sonogramas de la llamada de anuncio de E. patriciae. A, representación de cuatro llamadas de anuncio, se distinguen los armónicos; B, oscilograma; C, sonograma. Localidad: La Pirámide, Valle Nuevo (12 ${ }^{\circ}$; humedad relativa: 95 \%). Foto: Luis Díaz.

Los machos vocalizando se encontraron a una altura de $0-0.6$ metros $(\overline{\mathrm{x}}=0.4 \mathrm{~m})$ sobre el suelo. La planta más usada como percha para vocalizar fue el pajón, por lo que, las superficies más utilizadas durante las vocalizaciones fueron la parte superior de las plantas jóvenes, entre 0.5 y los $2.0 \mathrm{~m}$ del suelo, en las hojas de los pajones (70\%), seguido por las hojas de los helechos (15\%), las plantas de zarzamora (Rubus ulmifolius; $5 \%$ ), pinos jóvenes (Pinus occidentalis) ( $3 \%$ ) y en menor proporción herbáceas, gramíneas, musgos y arboles jóvenes (5\%), superficies como rocas y troncos también fueron utilizados por los individuos $(2 \%)$.

Los valores de temperatura fueron fluctuantes, obteniéndose $2.8{ }^{\circ} \mathrm{C}$ como la más baja durante la noche y hasta $39{ }^{\circ} \mathrm{C}$ como la más alta registrada durante el día (Fig. 4). Durante la temporada seca se observó una disminución importante de la actividad acústica en toda el área, lo que igualmente coincide con los valores de temperatura más bajos registrados 9.1-10.3 ${ }^{\circ} \mathrm{C}\left(\overline{\mathrm{x}}=9.6^{\circ} \mathrm{C}\right)$; en cambio, en la temporada lluviosa hubo un incremento de la actividad y un aumento de temperaturas $11.6-18.4^{\circ} \mathrm{C}\left(\overline{\mathrm{x}}=14.5^{\circ} \mathrm{C}\right)$. La velocidad del viento fluctuó entre 2 y $10 \mathrm{~km} / \mathrm{h}(\overline{\mathrm{x}}=3.4 \mathrm{~km} / \mathrm{h})$. 


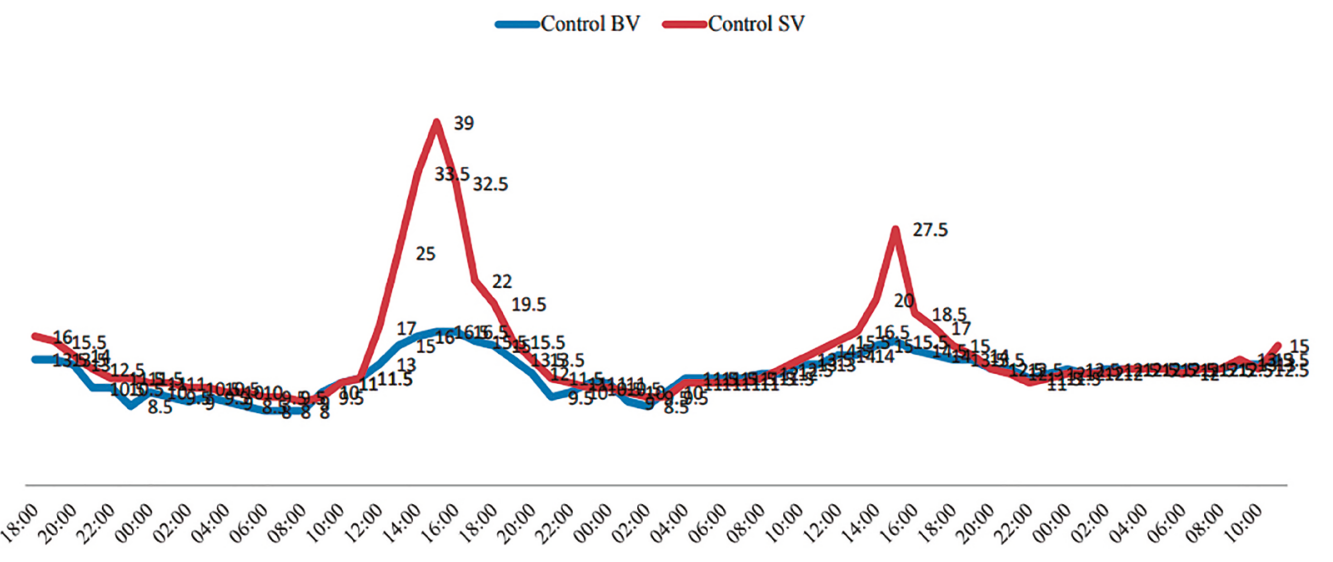

Figura 4. Comparación de la variación de temperatura durante dos días y tres noches en temporada lluviosa. Rojo, en el estrato sobre vegetación; azul, bajo vegetación.

\section{DISCUSIÓN}

La única especie que se encontró vocalizando junto a E. patriciae en las sabanas de pajón (Danthonia domingensis) fue E. haitianus. Esta última tiene una talla notablemente menor (machos con 12.4-15.5 $\mathrm{mm}$ de longitud hocico cloaca) y produce llamadas constituidas por largos trenes de 19-45 notas, los cuales tienen una duración de 3.6-5.8 segundos; la frecuencia dominante es más alta que en E. patriciae, 4.7-5.6 kHz (Díaz et al., 2018), lo que debe estar en relación con su tamaño. De manera que las llamadas de anuncio de ambas especies no son confundibles y existen notables diferencias, tanto espectrales como temporales, que sugieren una fuerte segregación en el nicho acústico (Fig. 5). De las especies dominicanas que se han caracterizado acústicamente, E. parabates es la única que emite llamadas de anuncio a manera de silbidos. En este caso, las llamadas tienen dos picos de modulación de amplitud, una tasa de repetición mayor (141 llamadas/minuto) y la frecuencia dominante es más alta $(2.6-2.8 \mathrm{kHz})$ que en E. patriciae (Díaz et al., 2018).
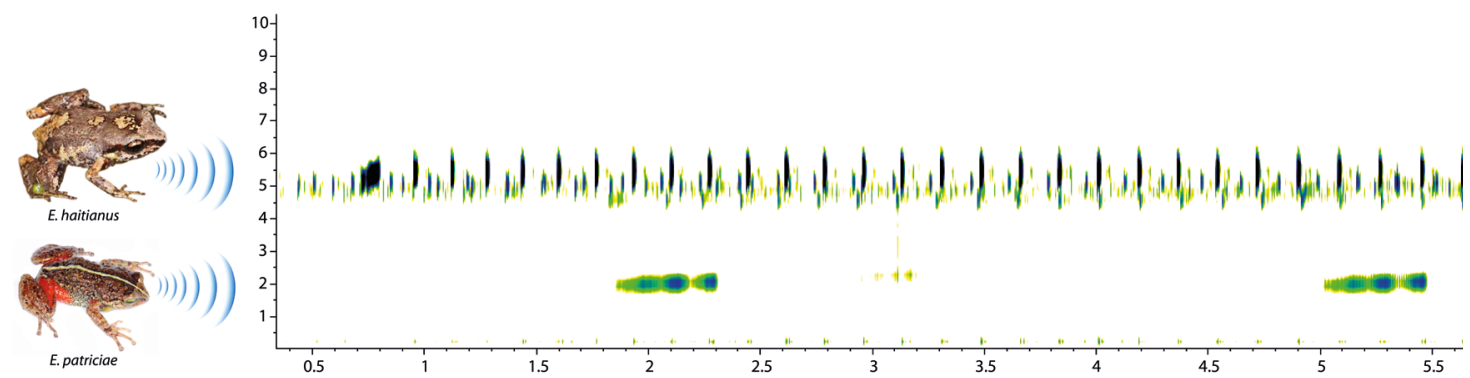

Figura 5. Sonograma de una grabación de E. haitianus y E. patriciae, en la localidad de La Pirámide, Valle Nuevo $\left(13^{\circ} \mathrm{C}\right.$; humedad relativa, $80 \%$ ). Se puede apreciar la segregación espectral entre ambas especies. 
En E. patriciae los armónicos se extienden al espectro ultrasónico ( $>20 \mathrm{kHz})$, al igual que fue registrado en $E$. brittoni por Ríos-Franceschi y Joglar (2016). De acuerdo con Akre et al. (2014), la producción de armónicos en las llamadas de Engystomops pustulosus (Cope) puede estar más relacionada con los procesos biomecánicos de las emisiones acústicas que con cualquier preferencia de las hembras por sonidos espectralmente más complejos. Algunas especies pequeñas de ranas emiten componentes de frecuencia en el espectro ultrasónico, lo cual no implica que estos tengan relevancia biológica (Köhler et al., 2017). En cambio, Narins et al. (2004) plantearon que la presencia de armónicos en la rana arborícola asiática Odorrana tormota $(\mathrm{Wu})$ permite que la señal emitida sobresalga en el entorno sonoro dominado por torrentes y cascadas, un hábitat totalmente diferente al de E. patriciae. Sin embargo, Feng y Narins (2008) y Shen et al. (2008) sugirieron que en O. tormota la producción de armónicos evolucionó hacia una audición diferencial de los machos, pero no desencadena respuestas por parte de las hembras. Todo lo anteriormente expuesto abre nuevas perspectivas para estudios de la comunicación acústica de E. patriciae, en que la emisión de armónicos puede o no tener relevancia para la comunicación de la especie.

\section{AGRADECIMIENTOS}

Al FONDOCYT, por el financiamiento para la realización del proyecto "Ecología asociada al suelo en la Sabana de pajón del Parque Nacional Juan Bautista Pérez Rancier (Valle Nuevo) y su respuesta a los impactos de la agricultura y fuego", este se llevó a cabo durante los años 2010-2012 a través del Museo Nacional de Historia Natural "Prof. Eugenio de Jesús Marcano", en coautoría con el Instituto de Investigaciones Botánicas y Zoológicas de la Universidad Autónoma de Santo Domingo. También al FONDOCYT por el proyecto "Caracterización y Ecología de la Flora y la Fauna en las sabanas de las Montañas Altas de la Cordillera Central y Sierra de Neiba" el cual se llevó a cabo a través del Instituto de Investigaciones Botánicas y Zoológicas de la Universidad Autónoma de Santo Domingo en coautoría con el Museo Nacional de Historia Natural "Prof. Eugenio de Jesús Marcano", durante los años 2014-2016. Contó, así mismo, con información de campo registrada en la base de datos sobre los anfibios de República Dominicana, producida con la participación de los autores. Estos datos fueron generados por el proyecto FONDOCYT "Anfibios en Peligro y Cambio Climático en la República Dominicana RANA RD". Se agradece igualmente a quienes brindaron apoyo durante el proyecto de tesis: Gabriel de los Santos, Carlos Suriel, Ruth Bastardo, Solanlly Carrero Jiménez, Alexis Hilario, América Sánchez Rosario, Robert Ortiz, Eveling Gabot, Arturo León, Francisco Paz y al cuerpo de guardaparques del Parque Nacional Valle Nuevo.

\section{LITERATURA CITADA}

Akre, K. L., X. Bernal, A. S. Rand y M. J. Ryan. 2014. Harmonic calls and indifferent females: no preference for human consonance in an anuran. Proceedings of the Royal Society B, 281: 20140986. http://dx.doi.org/10.1098/rspb.2014.0986

Cassá, C. 2012. Valle Nuevo: el parque Juan B. Pérez Rancier y su altiplano. Editora: Archivo General de la Nación (vol.CLXIV), ISBN:978-9945-074-54-3. Editora Búho, S.R.L. $229 \mathrm{pp}$. 
Díaz, L. M. y A. Cádiz. 2008. Guía taxonómica de los anfibios de Cuba. Abc TAXA, vol: 4. $294 \mathrm{pp}$.

Díaz, L. M., S. J. Incháustegui, C. Marte, G. Köhler, A. Cádiz y M. Rodríguez. 2018. Anew frog of the Eleutherodactylus abbotti species group (Anura: Eleutherodactylidae) from Hispaniola, with bioacoustic and taxonomic comments on other species. Novitates Caribaea, 12: 25-42.

Feng, A. S. y P. M. Narins. 2008. Ultrasonic communication in concave-eared torrent frogs (Amolops tormotus). Journal of Comparative Physiology A 194, 159-167. https://doi.org/10.1007/s00359-007-0267-1

Foden, W., B. Young, R. Akçakaya, R. Garcia, A. Hoffman, B. Stein, C. Thomas, C. J. Wheatley, D. Bickford, J. Carr, D. Hole, T. Martin, M. Pacifici, J. W. Pearce-Higgins, P. J. Platts, P. Visconti, J. Watson, B. Huntley. 2018. Climate change vulnerability assessment of species. WIREs Climate Change. e551. ISSN 1757-7799.

Frolich, L. M., N. Schultz, D. Almeida, y F. Nogales. 2003. Las Ranas de Los Andes Norte de Ecuador: Cordillera Oriental. Quito-Ecuador: Ediciones Abya Yala, 78 pp.

Galvis, P., A. V. Zaffaroni-Caorsi, S. J. Sánchez-Pacheco y M. Rada. 2016. The advertisement calls of three Eleutherodactylus species from Hispaniola (Anura: Eleutherodactylidae). Bioacoustics, $12 \mathrm{pp}$.

Granda-Rodríguez, H. D., A. del Portillo-Mozo y J. M. Renjifo. 2008. Uso de hábitat en Atelopus laetissimus (Anura: Bufonidae) en una localidad de la Sierra Nevada de Santa Marta, Colombia. Herpetotropicos, 4 (2): 87-93.

Guerrero, A. y M. M. McPherson. 2002. Historia Integrada de la región del Parque Nacional Juan Bautista Pérez Rancier (Valle Nuevo), 147 Pp. En: Evaluación Ecológica Integrada Parque Nacional Juan B. Pérez Rancier (Valle Nuevo), Editor: Francisco Núñez. Secretaria de Estado de Medio Ambiente y Recurso Naturales / Fundación Moscoso Puello, 80-90.

Hager, J. y T. Zanoni. 1993. La vegetación natural de la República Dominicana. Una nueva clasificación. Moscosoa, 7: 39-81.

Hedges, S. B., S. J. Incháustegui y R. Powell. 2004. Eleutherodactylus patriciae. In: IUCN 2008. 2008 IUCN Red List of Threatened Species. http://www.iucnredlist.org.

Hedges, S. B., W. E. Duellman y M. P. Heinicke. 2008. New World direct-developing frogs (Anura: Terrana): molecular phylogeny, classification, biogeography, and conservation. Zootaxa, 1737: 1-182.

Henderson, R. W. y R. Powell. 2009. Natural history of West Indian reptiles and amphibians. University press of Florida, Gainesville, $495 \mathrm{pp}$.

Köhler, J., M. Jansen, A. Rodríguez, P. J. R. Kok, L. F. Toledo, M. Emmrich, F. Glaw, C. F. B. Haddad, M. O. Rödel y M. Vences. 2017. The use of bioacoustics in anuran taxonomy: theory, terminology, methods and recommendations for best practice. Zootaxa, 4251 (1): 1-124. https://doi.org/10.11646/zootaxa.4251.1.1 
Ministerio de Medio Ambiente y Recursos Naturales. 2011. Lista de Especies de Fauna en Peligro de Extinción, Amenazadas o Protegidas de la República Dominicana (Lista Roja Nacional), Santo Domingo, República Dominicana, 44 pp.

Narins, P. M., A. S. Feng, W. Lin, H. U. Schnitzler, A. Denzinger, R. A. Suthers y C. Xu. 2004. Old World frog and bird vocalizations contain prominent ultrasonic harmonics. Journal of the Acoustical Society of America, 115 (2): 910-913. https://doi.org/10.1007/BF00612008

Núñez, F., N. Ramírez, M. McPherson y F. Portorreal. 2006. Plan de Conservación, Parque Nacional Juan Bautista Pérez Rancier (Valle Nuevo). Editora Amigo del hogar. Santo Domingo, República Dominicana, 87 pp.

Pough, H. F. 1999. Salamanders, anurans and caecilians. En: Pough, H. F., C. M. Janis y J. B. Heiser (eds.). Vertebrate Life. Fifth Edition, Prentice Hall, New Jersey, 773 pp.

Ríos-Franceschi, A. y R. L. Joglar. 2016. Harmonics: Rediscovering Eleutherodactylus Vocalizations. Life: The Excitement of Biology, 5 (4): 173-180.

Rueda, J. V. 1999. Anfibios y reptiles amenazados de extinción en Colombia. Revista de la Academia Colombiana de Ciencias Exactas, Física y Naturales, 23: 475-497.

Sangermano, F. L., P. Galvis, R. E. Gullison, J. Hardner y G. S. Ross. 2015a. Habitat suitability and protection status of four species of amphibians in the Dominican Republic. Elsevier Ltd, 55-65.

Sangermano, F. L., P. Galvis, R. E. Gullison, J. Hardner y G. S. Ross. 2015b. Forest baseline and deforestation map of the Dominican Republic through the analysis of time series of MODIS data. Elsevier Ltd, 363-367.

Schwartz, A. 1964. Two new species of Eleutherodactylus from the eastern Cordillera Central of the Dominican Republic. Caribbean Journal of Science, 4: 473-484.

Schwartz, A. y R. W. Henderson. 1991. Amphibians and reptiles of the West Indies. Descriptions, distributions and natural history. University of Florida Press. Gainesville, 720 pp.

Shen, J. X., A. S. Feng, Z. M. Xu, Z. L. Yu, V. S. Arch, X. J. Yu y P. M. Narins. 2008. Ultrasonic frogs show hyperacute phonotaxis to female courtship calls. Nature Letters, 453: 914-917. https://doi.org/10.1038/nature06719

Stuart, S. N., M. Hoffmann, J. S. Chanson, N. A. Cox, R. J. Berridge, P. Ramani y B. E.Young. (eds.) 2008. Threatened amphibians of the World. Lynx Edicions, Barcelona, Spain; IUCN, Gland, Switzerland; and Conservation International, Arlington, Virginia, USA, 758 pp.

Unión Internacional para la Conservación de la Naturaleza (UICN). 2014. IUCN Red List of Threatened Species. Versión 2014. 3. http://www.iucnredlist.org. Última actualización 30 de mayo del 2017. 
Urbina-Cardona, J. N. y J. Pérez-Torres. 2002. Dinámica y preferencias de microhabitats en dos especies del género Eleutherodactylus (Anura: Leptodactylidae) de bosque andinos, $278-285$.

Wells, K. D. 2007. The ecology and behavior of amphibians. The University of Chicago Press. Chicago, Illinois, U.S.A.

Cómo citar: Marte-Pimentel, C. F., Díaz, L. M., \& Incháustegui, S. J. (2022). Descripción preliminar de las llamadas de anuncio de Eleutherodactylus patriciae (Anura: Eleutherodactylidae), en el Parque Nacional Valle Nuevo, República Dominicana. Novitates Caribaea, (19), 1-11. https://doi.org/10.33800/nc.vi19.286. Artículo científico original. 